\title{
Rubber friction on (apparently) smooth lubricated surfaces
}

\author{
M. Mofidi, B. Prakash \\ Division of Machine Elements, Lulea University of Technology, Lulea SE-97187, Sweden \\ B.N.J. Persson \\ IFF, FZ-Jülich, 52425 Jülich, Germany \\ O. Albohr \\ Pirelli Deutschland AG, 64733 Höchst/Odenwald, Postfach 1120, Germany
}

\begin{abstract}
We study rubber sliding friction on hard lubricated surfaces. We show that even if the hard surface appears smooth to the naked eye, it may exhibit short wavelength roughness, which may give the dominant contribution to rubber friction. That is, the observed sliding friction is mainly due to the viscoelastic deformations of the rubber by the substrate surface asperities. The presented results are of great importance for rubber sealing and other rubber applications involving (apparently) smooth surfaces.
\end{abstract}

\section{Introduction}

Rubber friction on smooth surfaces is a topic of great practical importance, e.g., for rubber sealing, wiper blades or for the contact between a tire and the metal rim[1]. For perfectly smooth surfaces rubber friction is believed to be due to periodic cycles of pinning, elastic deformation, and rapid slip of rubber molecules [2, 3, 4] or, more likely, small patches [5] of the rubber at the sliding interface. In a recent publication, Vorvolakos and Chaudhury [6] (see also Ref. [7, 8]) have studied rubber friction for a silicone elastomer sliding on extremely smooth Si wafer, with the root-mean-square roughness $\approx$ $0.5 \mathrm{~nm}$, covered by inert self-assembled monolayer films. The observed friction as a function of the sliding velocity exhibit a bell-like shape as expected from theory 2, 5]. However, a surface which appears smooth to the naked eye may exhibit strong surface roughness at short length scales, e.g., at the micrometer and nanometer length scale. This is true even for highly polished surfaces which may appear perfectly smooth to the naked eye. When a rubber block slides on a hard surface with surface roughness, a large contribution to the friction force may arise from the time-dependent, substrate asperity-induced deformations of the rubber surface. That is, during sliding the substrate asperities give rise to pulsating deformations of the rubber, which will result in energy dissipation because of the internal friction of the rubber. This is believed to be the major contribution to the tire-road friction [9, 10]. In this paper we will show that the roughness of a highly polished steel surface may also give the dominant contribution to the friction, even for lubricated surfaces. This result is very important for rubber sealing applications [11], in particular at low sliding velocities and low temperatures.

\section{Rubber friction: experimental results}

Friction tests have been carried out using a reciprocating tribometer where a steel cylinder (diameter $D=1.5 \mathrm{~cm}$ and length $L=2.2 \mathrm{~cm}$ ) is squeezed against the substrate (rubber block, thickness $4 \mathrm{~mm}$ ), see Fig. 1. The steel cylinder performs longitudinal oscillations against the rubber block with a stroke $a=1 \mathrm{~mm}$ and frequency $f=50 \mathrm{~Hz}$. This gives the average slip velocity $v \approx 0.1 \mathrm{~m} / \mathrm{s}$. The rubber specimens [acronitrile butadiene rubber (NBR)] have been washed in industrial petroleum for 3 minutes by using an ultrasonic cleaner and then dried for 10 minutes. The rubber surface has the root-mean-square roughness $\approx 0.4 \mu \mathrm{m}$, and has parallel grooves caused during molding of elastomer sheets in steel mold. The steel cylinder has a root-mean-square roughness of $\approx 0.1 \mu \mathrm{m}$.

Fig. 2 shows the power spectrum of the surface roughness of the steel surface. The power spectrum is defined by 12 ]

$$
C(q)=\int d^{2} x\langle h(\mathbf{x}) h(\mathbf{0})\rangle e^{i \mathbf{q} \cdot \mathbf{x}}
$$

where $\langle.$.$\rangle stands for ensemble averaging. Here h(\mathbf{x})$ is the surface height at the point $\mathbf{x}$, where we have assumed $\langle h(\mathbf{x})\rangle=0$. The surface height was measured over different surface areas using Atomic Force Microscopy and an optical method, and Fig. 2 was obtained from three different measurements involving different resolution. The straight (green) line has a slope corresponding to the fractal dimension $D_{\mathrm{f}} \approx 2.66$. In the calculations of the friction presented below we have used the this linear approximation and included the surface roughness power spectra over the full wave vector range shown in the figure. Thus the longest and the shortest wavelength roughness included in the analysis is $\lambda_{0}=2 \pi / q_{0} \approx 0.3 \mathrm{~mm}$ and $\lambda_{1}=2 \pi / q_{1} \approx 6 \mathrm{~nm}$.

The experimental results presented in Fig. 5 and 6 were obtained for the load $F_{\mathrm{N}}=100 \mathrm{~N}$ and with a test duration of $15 \mathrm{~min}$. Since the oscillation stroke is very small $(1 \mathrm{~mm})$ one expects that most of the oil is squeezed out from the steel cylinder - rubber contact region.

The viscoelastic modulus $E(\omega)$ has been measured (using Eplexor 150) using a rectangular rubber block 


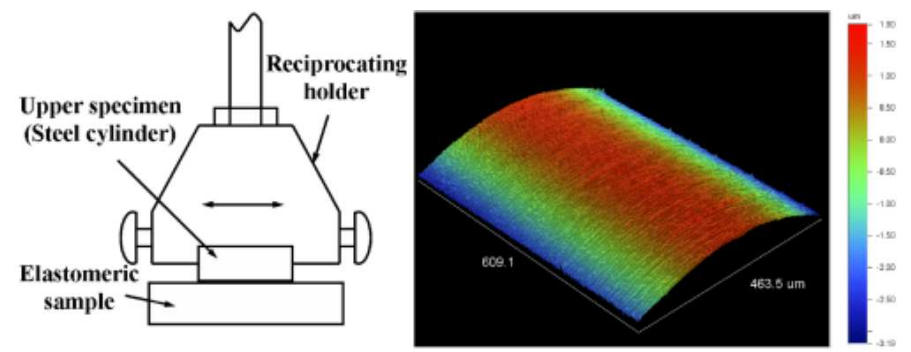

FIG. 1: Test configuration for friction studies under reciprocating sliding conditions.

$5 \times 2 \times 30 \mathrm{~mm}^{3}$. The measurements were done in tension with $8 \%$ of prestrain and $1.3 \%$ of dynamic strain amplitude. Fig. 3 shows the logarithm of the real part of the viscoelastic modulus of the acronitrile butadiene rubber used in the present study, as a function of the logarithm of the frequency $\omega$, for the temperatures $T=50{ }^{\circ} \mathrm{C}$ and $80{ }^{\circ} \mathrm{C}$.

The diameter $d$ of the contact region between the steel cylinder and the rubber substrate can be estimated using the Hertz contact theory for bodies with cylinder geometry, see Fig. 4. For elastic solids, the diameter $d$ of the contact area is given by 13$]$

$$
d=2\left(\frac{2 F_{\mathrm{N}} D}{\pi L E^{*}}\right)^{1 / 2},
$$

where $E^{*}=E /\left(1-\nu^{2}\right)$ (where $E$ is the Young modulus and $\nu$ the Poisson ratio). The average pressure in the contact region is

$$
\bar{p}=\frac{1}{2}\left(\frac{\pi F_{\mathrm{N}} E^{*}}{2 L D}\right)^{1 / 2} .
$$

For $F_{\mathrm{N}}=100 \mathrm{~N}$ and for $T \approx 50{ }^{\circ} \mathrm{C}$ we have (see Fig. 3 ) $E^{*} \approx 10 \mathrm{MPa}$ (where we have assumed the frequency $\omega \approx$ $10^{-3} \mathrm{~s}^{-1}$, corresponding to the contact time $\sim 1000 \mathrm{~s}$ ) giving $d \approx 0.4 \mathrm{~cm}$ and $\bar{p} \approx 1 \mathrm{MPa}$.

Fig. 5 shows the measured friction coefficients for the steel cylinder sliding against non-aged rubber in 11 different lubrication oils with very different viscosities. Thus, for example, the PA01 and PA02 oils have the viscosities (at $T=40{ }^{\circ} \mathrm{C}$ ) $4.4 \times 10^{-3}$ and $22.8 \times 10^{-3} \mathrm{~Pa} \mathrm{~s}$, respectively. In spite of the large difference in viscosities, the rubber friction coefficients are nearly equal. This indicates that the rubber friction is not (mainly) due to shearing a thin viscous layer, but due to the internal friction of the rubber (see below).

Fig. 6] shows the measured friction coefficients for aged rubber. The aged rubber samples were prepared by immersing them in different base fluids at $T=125^{\circ} \mathrm{C}$ for one week. NBR rubber has polar nitrile groups and nonpolar oils such as naphthenic have nearly no effect on the properties of NBR rubber, and this explained why rubber aged in naphthenic exhibits nearly the same friction as for

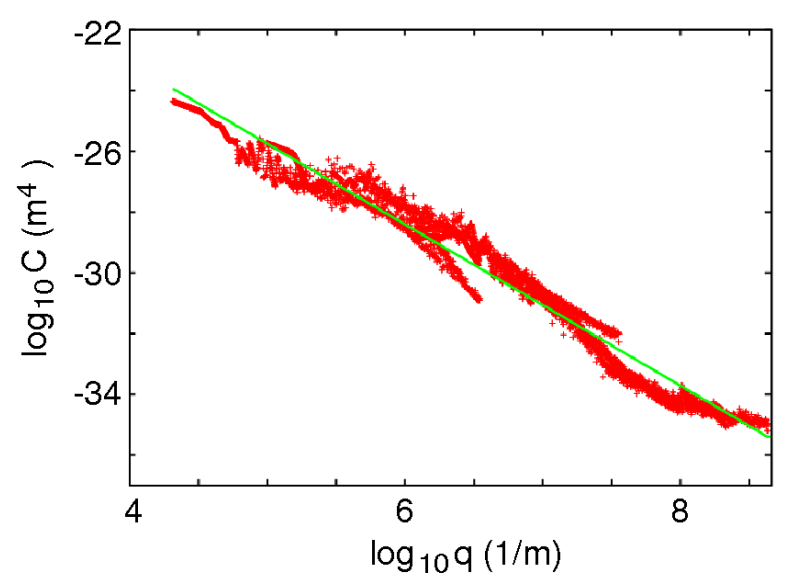

FIG. 2: The power spectrum of the surface roughness of the steel surface. The root-mean-square surface roughness is about $0.08 \mu \mathrm{m}$. The straight line has a slope corresponding to the fractal dimension $D_{\mathrm{f}} \approx 2.66$.

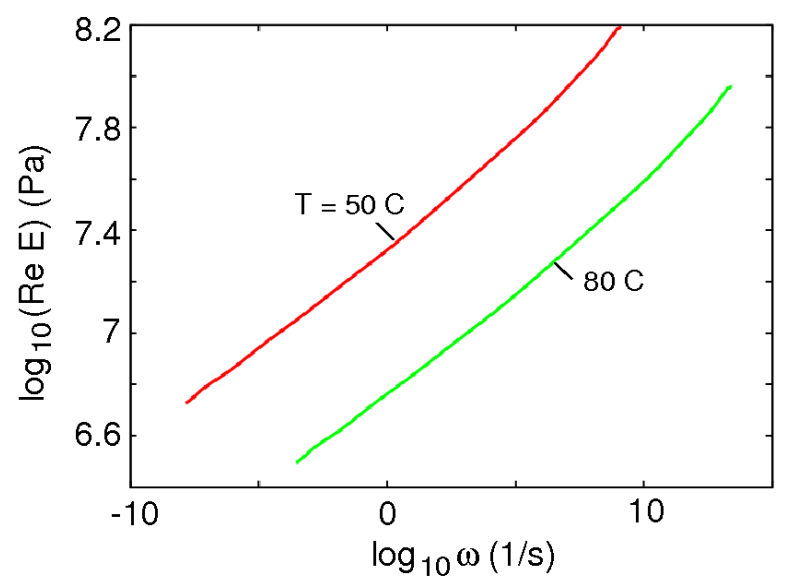

FIG. 3: The logarithm of the real part of the viscoelastic modulus as a function of the logarithm of the frequency $\omega$ for the temperatures $T=50{ }^{\circ} \mathrm{C}$ and $80{ }^{\circ} \mathrm{C}$. For acronitrile butadiene rubber.

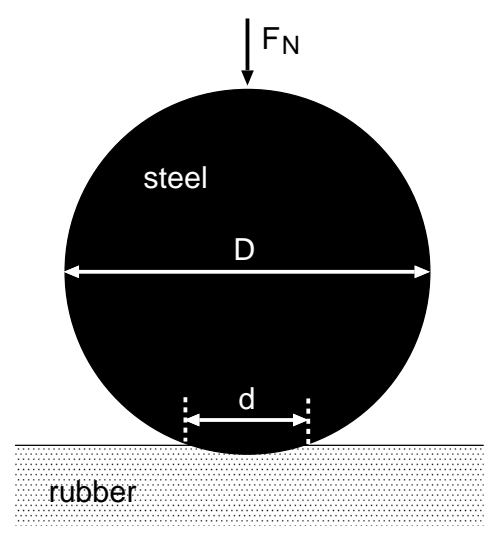

FIG. 4: Steel cylinder squeezed in contact to a rubber substrate. 


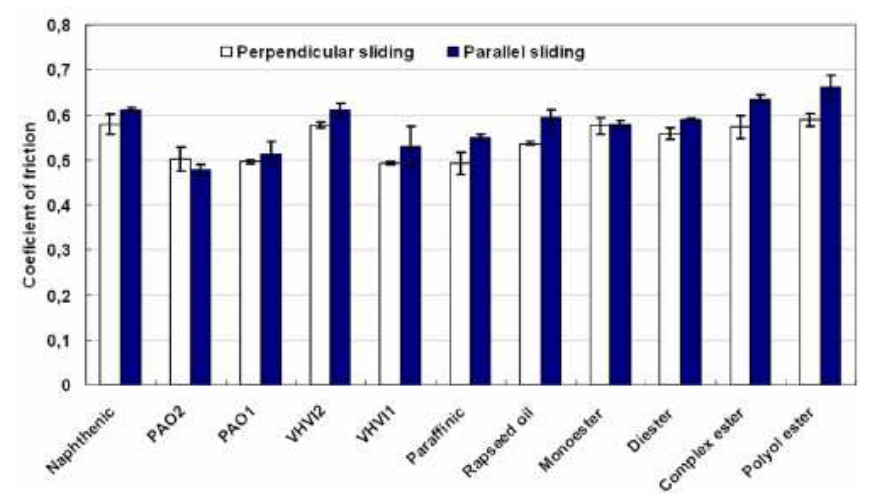

FIG. 5: Coefficient of friction of non-aged samples in different base oils. For the load $F_{\mathrm{N}}=100 \mathrm{~N}$.

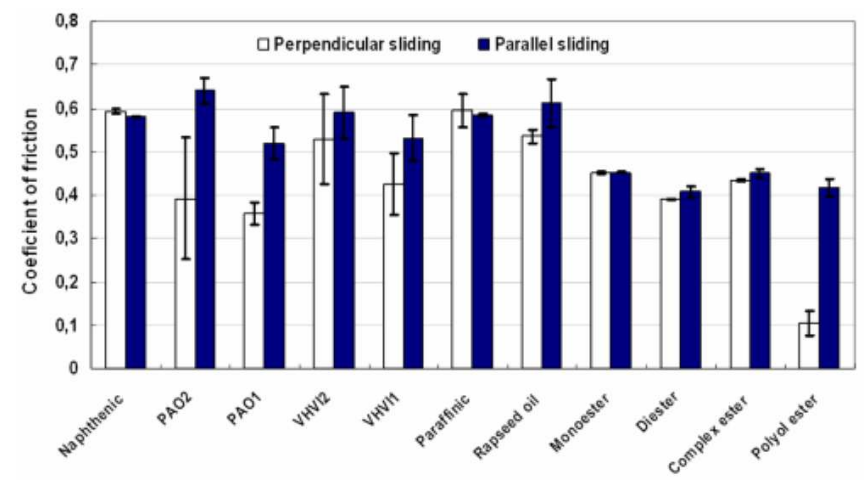

FIG. 6: Coefficient of friction of aged samples in different base oils. For the load $F_{\mathrm{N}}=100 \mathrm{~N}$.

non-aged NBR rubber (compare Fig. 5 with 6 ). However, oils with polar groups, e.g. polyol ester, will diffuse into the rubber which may reduce the internal friction of the rubber. In addition, when the rubber block is squeezed against the substrate, oil may be squeezed out from the rubber matrix, giving a thicker oil film at the interface and thus lower the friction (a similar effect is believed

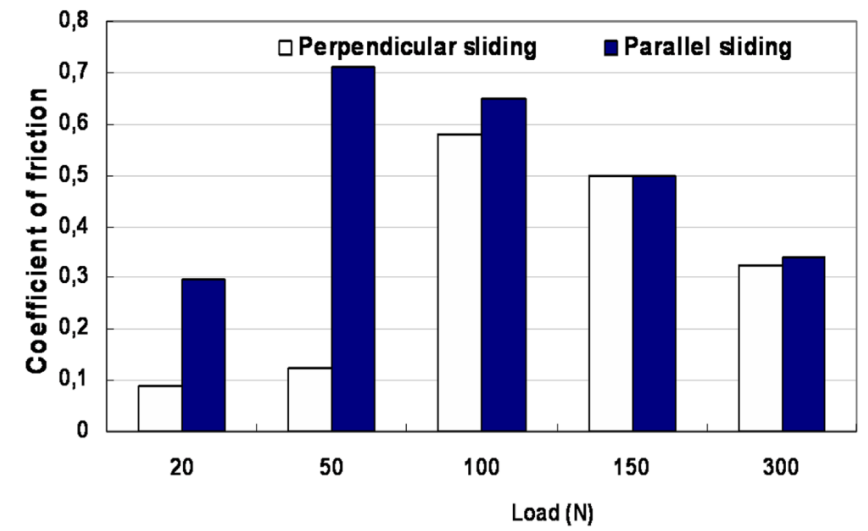

FIG. 7: Friction coefficient as a function of load at the background temperature $T=25^{\circ} \mathrm{C}$.

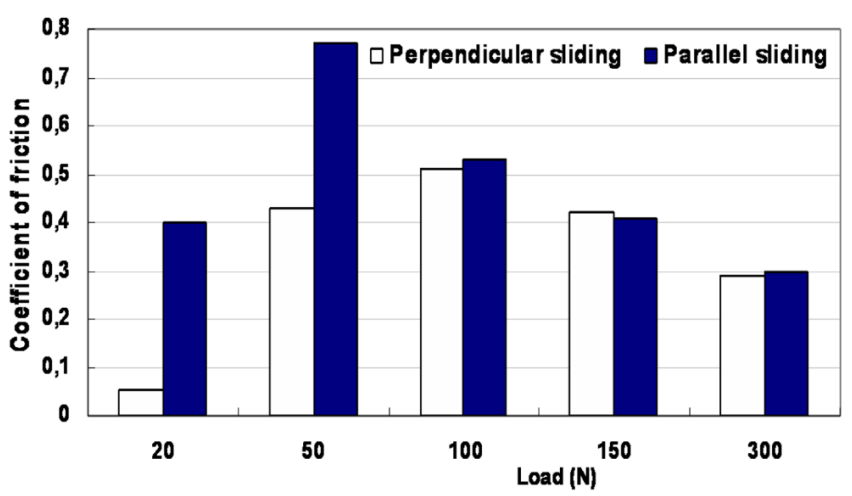

FIG. 8: Friction coefficient as a function of load at the background temperature $T=40{ }^{\circ} \mathrm{C}$.

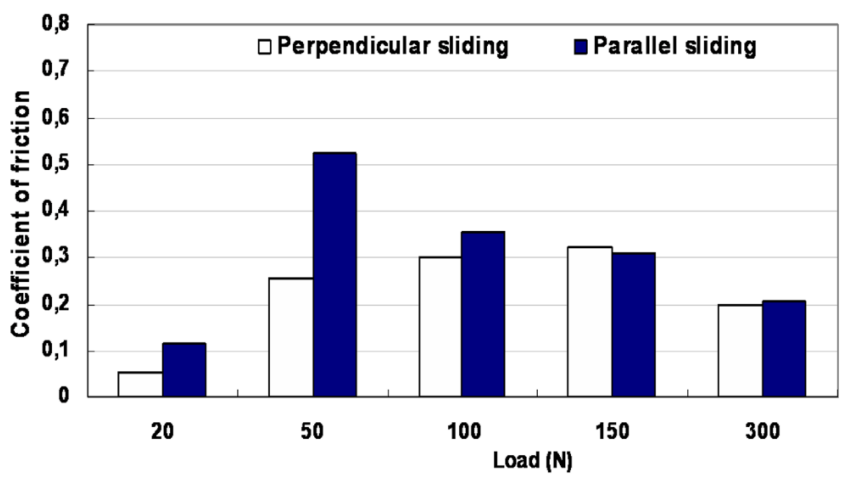

FIG. 9: Friction coefficient as a function of load at the background temperature $T=80{ }^{\circ} \mathrm{C}$.

to contribute to the extremely low friction exhibited by human joints [17]). We believe that both effects may contribute to why NBR rubber aged in polyol ester exhibits much smaller friction than the non-aged rubber.

Figures 7,8 and 9 show the friction coefficient for different loads and temperatures. Here the temperature refers to the background temperature, which was varied

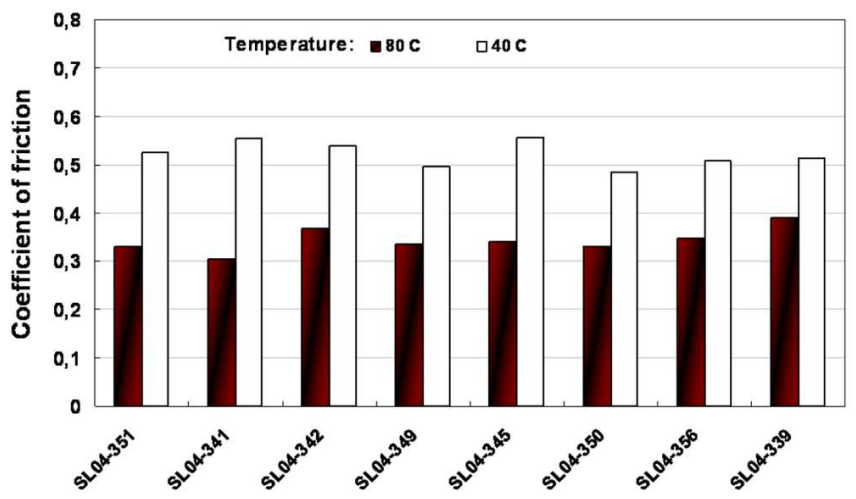

FIG. 10: Friction coefficient for one base oil with several different additives and for $T=40{ }^{\circ} \mathrm{C}$ and $80{ }^{\circ} \mathrm{C}$. For the load $F_{\mathrm{N}}=100 \mathrm{~N}$. 
by contacting the back-side of the rubber block to a metal block with the given temperature. (The temperature in the sliding contact is not known, but will be higher due to the frictional heating.) Note that as the temperature increases the friction decreases. This cannot result from the change in viscosity of the lubricant oil since we already know from above that the lubricant viscosity has a negligible influence on the friction, at least for the squeezing force $F_{\mathrm{N}}=100 \mathrm{~N}$, see Fig. 5. However, we will show in Sec. 3 that the temperature dependence of the sliding friction can be understood from the temperature dependence of the internal friction of the rubber. Thus, when the temperature increases the rubber becomes more elastic (less viscous) and the internal friction decreases.

The dependence of the rubber friction on the load can be understood as follows. For very small load $\left(F_{\mathrm{N}}=\right.$ $20 \mathrm{~N}$ ) the average pressure in the contact area [see Eq. (3)] is relative low and the grooves on the rubber surface will not be (fully) elastically flattened, and will trap lubricant oil, which may be pulled into the contact area during each oscillation. This will result in an oil film which is thick enough to reduce the rubber-steel asperity contact and hence lower the viscoelastic contribution to the friction. This drag of lubricant fluid into the contact area is particularly large when the oscillation direction is perpendicular to the grooves on the rubber surface[14], and this explains why the friction for small load is much lower for perpendicular sliding than parallel sliding. However, for high load $\left(F_{\mathrm{N}} \geq 100 \mathrm{~N}\right)$ there is negligible difference between parallel and perpendicular sliding, indicating that the lubricant has a negligible direct influence on the friction.

The drop in the friction for large load is most likely due to the increase in the temperature caused by the frictional heating. This effect becomes more important as the load increases, and explains why the friction decreases for high load. At lower sliding velocity (or oscillation frequency) the heating effects become less important (because of heat diffusion) and in this case one expects a smaller drop in the friction coefficient with increasing load. We plan to test this prediction experimentally.

Fig. 10 shows the friction coefficients (for the load $F_{\mathrm{N}}=100 \mathrm{~N}$ ) at $T=40{ }^{\circ} \mathrm{C}$ and $80{ }^{\circ} \mathrm{C}$ for the same base oil but with different additives. As expected, there is negligible dependence of the friction on the additives. The reason for this is the same as before: the observed friction is mainly due to the internal friction of the rubber which does not change between the different experiments. That is, although the additives in the base oil may adsorb on the solid surfaces and act as boundary lubricants, the result of the study above indicates that such (mono) layers have negligible influence on the friction.

\section{Rubber friction: theory}

We have calculated the dependence of the rubber friction on the sliding velocity and the temperature using the theory presented in Ref. [9]. The theory assumes that the friction is entirely due to the viscoelastic deformation of the rubber, which results from the pulsating deformations from the substrate asperities. The only inputs in the calculations are the surface roughness power spectrum (see Fig. 2) and the rubber viscoelastic modulus. We have measured the viscoelastic modulus $E(\omega)$ of the rubber as a function of frequency (and temperature). In the calculations we do not take into account the lubrication oil directly (but it influences the friction indirectly by reducing (or removing) the adhesion between the solid walls [15]). We have assumed the nominal contact pressure of $1 \mathrm{MPa}$.

Fig. 11 shows the steady state kinetic friction coefficient calculated using the measured surface roughness power spectrum (from Fig. 22) and the measured viscoelastic modulus of the rubber. Results are presented for the background temperatures $50^{\circ} \mathrm{C}$ and $80^{\circ} \mathrm{C}$. Note that the magnitude of the calculated friction coefficient at the sliding velocity $\sim 0.1-1 \mathrm{~m} / \mathrm{s}$ is similar to what is observed experimentally, and also the temperature dependence is in good agreement with the measurements (see Sec. 2).

In Fig. 12 we show (a) the friction coefficient $\mu_{\mathrm{k}}$, and (b) the logarithm of the (normalized) contact area $A / A_{0}$, as a function of the logarithm of the large-wavevector cut-off $q_{1}$ (in the calculations we only include surface roughness with wavevectors $q_{0}<q<q_{1}$ ). Results are presented for two different temperatures $T=50{ }^{\circ} \mathrm{C}$ and $80^{\circ} \mathrm{C}$ and for the sliding velocity $v=1 \mathrm{~m} / \mathrm{s}$. The figure shows that the long-wavelength roughness gives a negligible contribution to the friction. The reason for why only the short-wavelength roughness is important in the present case is the large fractal dimension $\left(D_{\mathrm{f}} \approx 2.7\right)$ of the steel surface, which implies that the ratio between the amplitude and the wavelength of the surface roughness strongly increases as the wavelength decreases [16], and this makes the short-wavelength roughness much more important than the long-wavelength roughness.

\section{Squeeze-out}

We have argued above that the observed rubber friction can be explained as resulting from the viscoelastic deformations of the rubber by the countersurface asperities. In this section we briefly address the role of the lubrication oil. We first note that the oil will effectively eliminate the adhesive interaction between the rubber and the countersurface [15]. Most of the oil will be squeezed out from the steel-rubber contact area, but a molecular thin layer may remain even after long squeezing time.

Consider first a flat rigid rectangular block squeezed against a flat hard countersurface with the nominal (or average) pressure $p$ in a lubrication fluid with the viscosity $\eta$. The separation between the surfaces after the time 


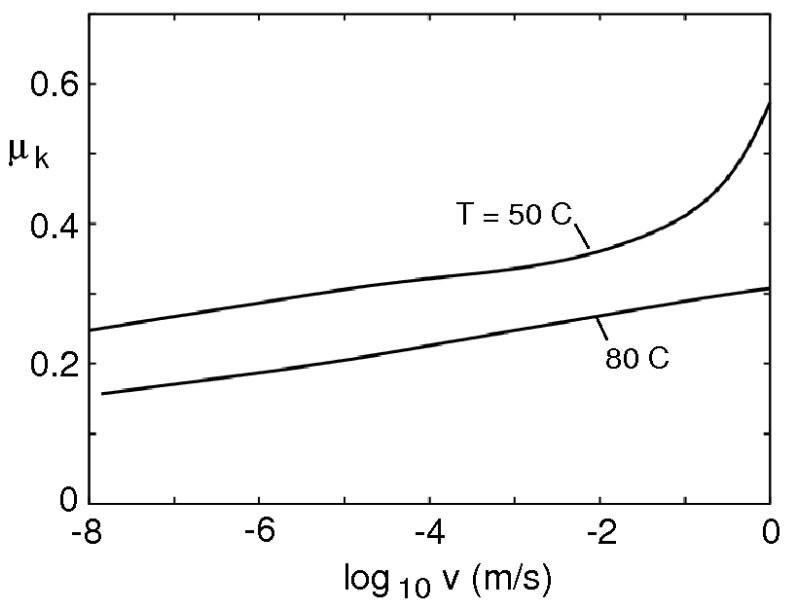

FIG. 11: The steady state kinetic friction coefficient calculated using the measured surface roughness power spectrum (from Fig. 2) and the measured viscoelastic modulus of the rubber. For the background temperatures $50^{\circ} \mathrm{C}$ and $80^{\circ} \mathrm{C}$, and the nominal squeezing pressure $p=1 \mathrm{MPa}$.

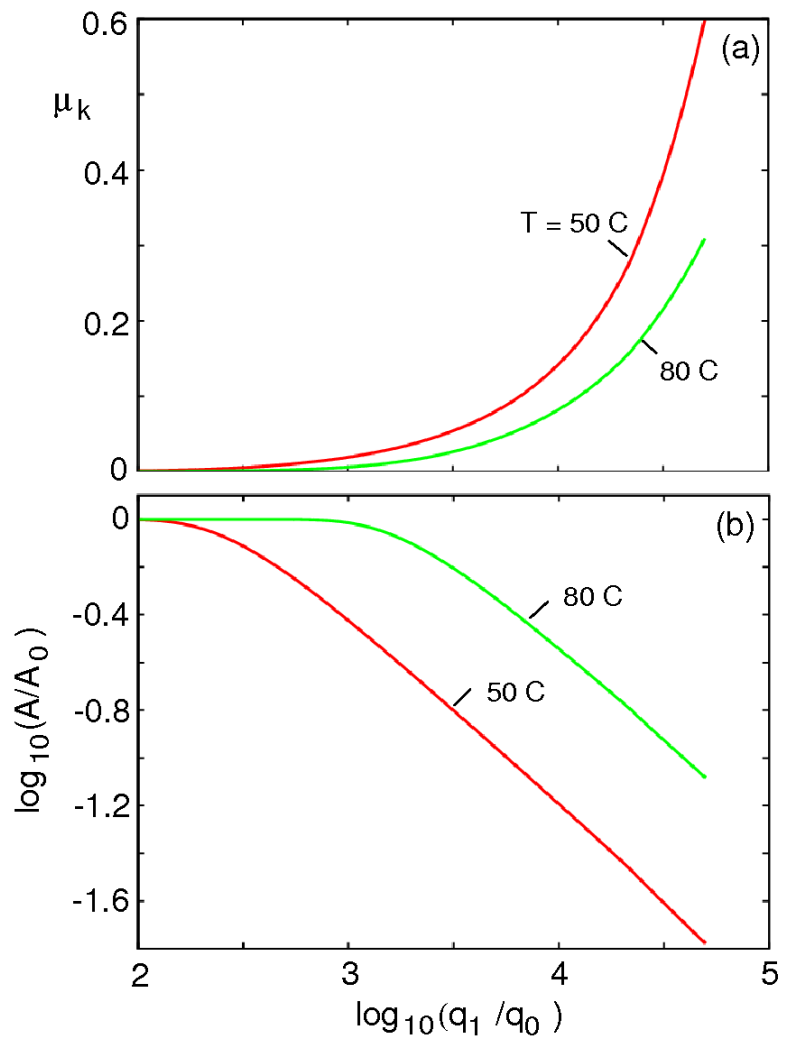

FIG. 12: The friction coefficient $\mu_{\mathrm{k}}$ (a) and the logarithm of the (normalized) contact area $A / A_{0}(\mathrm{~b})$, as a function of the logarithm of the large-wavevector cut-off $q_{1}$ [in units of the low-wavevector cut-off $\left.q_{0}\right]$. In the calculations we only include surface roughness with wavevectors $q_{0}<q<q_{1}$. Results are presented for two different temperatures $T=50{ }^{\circ} \mathrm{C}$ and $80{ }^{\circ} \mathrm{C}$ and for the sliding velocity $v=1 \mathrm{~m} / \mathrm{s}$. $t$ is (see Fig. 13) 17]

$$
h(t) \approx(\eta / 2 p t)^{1 / 2} d .
$$

Here $d$ is the width of the bottom surface of the block and we assume that $d<<L$, where $L$ is the length of the bottom surface of the block. With $d \approx 0.4 \mathrm{~cm}, p \approx 1 \mathrm{MPa}$ and with $t=1000 \mathrm{~s}$ we get with the typical viscosity $\eta \approx 0.01 \mathrm{~Pa} \mathrm{~s}, h(t) \approx 4 \mathrm{~nm}$. For surfaces with roughness the squeeze-out from asperity contact regions is even faster, but in this case some liquid may get "trapped" in sealed off regions [18]. For non-aged rubber the trapped islands may disappear because of diffusion of lubricant oil into the rubber matrix, see Fig. 14. The shear stress developed in a fluid film with thickness $h$ is $\sigma=\eta v / h$. In the present case, if $v=0.1 \mathrm{~m} / \mathrm{s}$ and $h=10 \mathrm{~nm}$ we get $\sigma=0.1 \mathrm{MPa}$ which would give a contribution to the friction coefficient of order $\sigma / p \approx 0.1$. However, the thickness of the oil film will be very non-uniform, and in many regions (cavity regions) at the interface the film may be much thicker than $10 \mathrm{~nm}$ (see below), and shearing the lubricant film in these regions will give a negligible contribution to the friction. In other regions, where the steel asperities make direct contact with the rubber, the local squeezing pressure is much higher than the average pressure, and in these regions at most a few monolayers of oil film will remain trapped. Nevertheless, since the region of direct wall-wall contact is only a small fraction of the nominal contact area, the contribution to the friction from shearing the confined thin layers appears to be negligible (see Sec. 2).

Fig. [15 shows the probability distribution $\bar{P}_{u}$ of surface separations $u$. This function has been calculated as outlined in Ref. [19]. In the calculation we have assumed a rubber elastic modulus $E=100 \mathrm{MPa}$ which correspond to the temperature $T=40{ }^{\circ} \mathrm{C}$ and the perturbing frequencies $\omega \approx 10^{6} \mathrm{~s}^{-1}$ (see Fig. 2), which is a typical perturbing frequency $(\omega=q v)$ from surface roughness with wavevector $q=10^{7} \mathrm{~m}^{-1}$ and sliding velocity $v=0.1 \mathrm{~m} / \mathrm{s}$. In the calculation we have neglected the direct influence of the lubrication oil, but it is accounted for indirectly by neglecting the adhesive interaction between the rubber and the steel surface. Using $\bar{P}_{u}$ we can give a more accurate estimate of the contribution from the oil film to the shear stress. We get the viscous shear stress

$$
\sigma \approx \eta v \int_{u_{c}}^{\infty} d u \frac{\bar{P}_{u}}{u}
$$

where $u_{\mathrm{c}}$ is a cut-off length of order $\sim 1 \mathrm{~nm}$ since molecular thin lubrication films cannot be described by the continuum theory of fluid mechanics 18]. We note that $\bar{P}_{u}$ has a delta function at the origin $u=0$, but in the present case this carry the weight $A\left(\zeta_{1}\right) / A_{0} \approx 0.01$ and the contribution from the area of real contact to the friction force can be neglected. Using the calculated $\bar{P}_{u}$ (see Fig. 15), and assuming $\eta=0.01 \mathrm{~Pa} \mathrm{~s}$ and $v=0.1 \mathrm{~m} / \mathrm{s}$, 


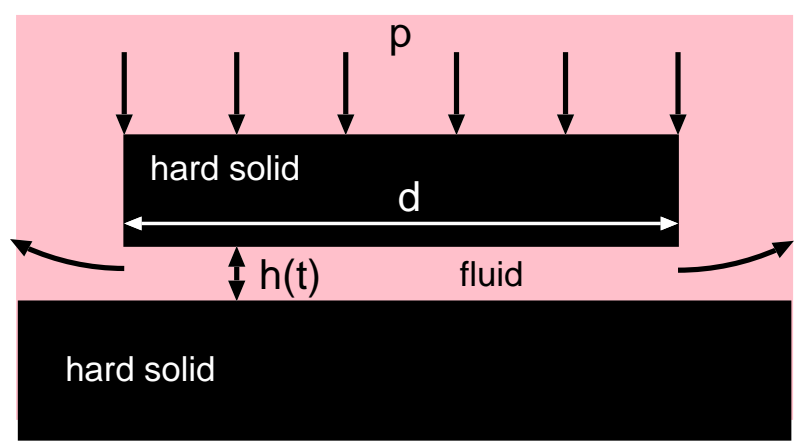

FIG. 13: A block squeezed against a substrate in a fluid. The separation between the bottom surface of the block and the top surface of the substrate is denoted by $h(t)$.

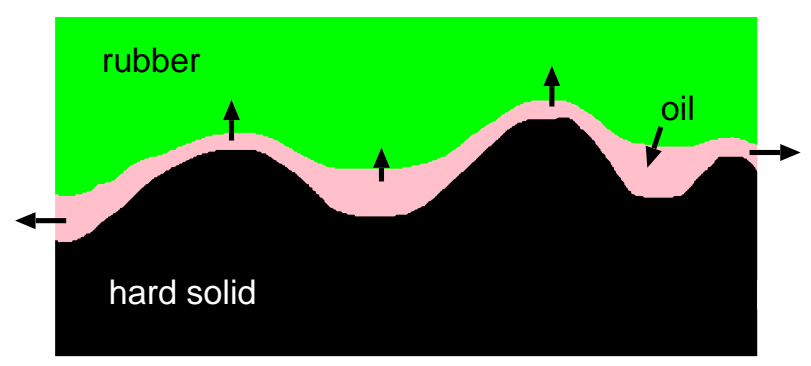

FIG. 14: A rubber block squeezed against a substrate in an oil. The oil is partly squeezed out at the external boundaries of the nominal contact area and partly transfered to (or from) the rubber matrix.

Eq. (5) gives $\sigma \approx 0.06 \mathrm{MPa}$ so the contribution to the friction from the lubricant film is very small, of order 0.06 (where we have assumed the normal stress $p=1 \mathrm{MPa}$ ). We note that this is likely to be an overestimation of the contribution of the oil film to the friction coefficient, as the oil film may tend to slightly increase the separation between the walls, and also because we have not accounted for the roughness on the rubber surface in the analysis.

\section{Discussion}

When a block of a viscoelastic solid, such as rubber, is sliding on a hard rough countersurface, the largest contribution to the sliding friction is usually derived from the time-dependent deformations of the rubber by the countersurface asperities. This is the case, for example, for the tire-road contact. Here we have shown that even a highly polished countersurface, which may appear mirror-smooth to the naked eye, may have enough surface roughness at short length scale to give a large contribution to rubber friction. This result has many important applications, e.g., in the context of rubber sealing.

In many rubber sealing applications the rubber surface

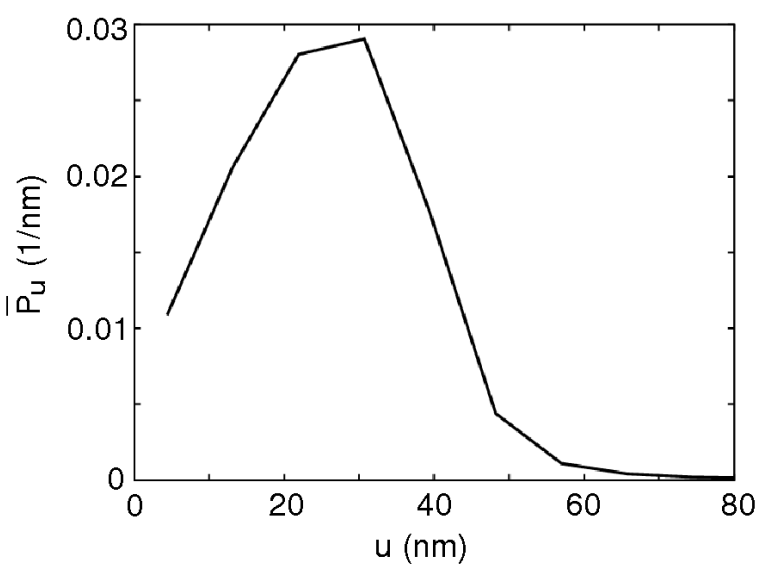

FIG. 15: The calculated probability distribution $\bar{P}_{u}$ of surface separations $u$.

and the (lubricated) countersurface are squeezed together for a long time between the slip events. Furthermore, during the onset (and stop) of sliding the slip velocities will be very small. This may result in nearly complete squeeze-out of the lubricant film. Thus, at some point in time slip will occur between what is effectively unlubricated surfaces. This may result in high friction and large wear, and perhaps failure of the seal with potentially serious consequences.

Note that with respect to sliding friction there is an asymmetry between roughness on the countersurface and on the rubber block. Thus, only roughness on the hard countersurface will contribute to the friction force. Roughness on the rubber surface may in fact lower the sliding friction by trapping lubrication fluid. On the other hand, with respect to stationary contact mechanics, roughness on the two surfaces plays a similar role [13, 20].

There is an important difference between rubber friction on very rough surfaces, such as a road surface, and rubber friction on smoother surfaces with only shortwavelength roughness. On very rough surfaces, as the magnification increases we observe smaller and smaller rubber-countersurface asperity contact regions, and the local stress and temperature will rapidly increase until the limit of strength of the rubber has been reached. For tread rubber in contact with road surfaces this limit is reached at the length scale (or resolution) $\lambda_{\mathrm{c}} \approx 1-10 \mu \mathrm{m}$, and at this length scale during slip strong wear processes occur. The rubber friction on road surfaces can be explained by including the the viscoelastic deformations of the rubber from road surface roughness with wavelength down to $\lambda_{\mathrm{c}}$. On the other hand, for surfaces with mainly short-wavelength roughness, such as the steel surface used in the present study, it may be necessary to include roughness with wavelength down to the molecular length scale, e.g., the distance between cross links in the rubber which typically is of order a few nanometers. This 
may result in different wear mechanisms and wear rates than on surfaces with large long wavelength roughness.

The results presented in this paper may also be relevant for the adhesion and locomotion of some animals on rough substrates. Thus, some animals, such as grasshoppers and tree frogs, have smooth attachment pads built from a (non-compact) material which is highly viscoelastic (like rubber) [21]. Furthermore, the toe pad-substrate contact region is wet (lubricated) with a liquid injected into the contact area by the animal. The liquid viscosity, the nominal squeezing pressure, and the size and shape of the contact area differ from the lubricated rubbersubstrate contact problem studied above, but some of the results presented above may nevertheless be relevant for the animal toe pad-substrate interaction problem 22, 23].

\section{Summary and conclusion}

We have presented a combined experimentaltheoretical study of rubber sliding friction against hard lubricated surfaces. We have shown that even if the hard surface appears smooth to the naked eye, it may exhibit short wavelength roughness, which may give the dominant contribution to rubber friction. The presented results may be of great importance for rubber sealing and other rubber applications involving (apparently) smooth surfaces.

[1] D. F. Moore, The Friction and Lubrication of Elastomers (Oxford: Pergamon, 1972)

[2] A. Schallamach, Wear 6, 375 (1963); A. Schallamach, Wear 17, 301 (1971).

[3] Y.B. Chernyak and A.I. Leonov, Wear 108, 105 (1986).

[4] A.E. Filippov, J. Klafter and M. Urbakh, Phys. Rev. Lett. 92, 135503 (2004).

[5] B.N.J. Persson and A.I. Volokitin, Eur. Phys. J. E21, 69 (2006).

[6] K. Vorvolakos and M.K. Chaudhury, Langmuir 19, 6778 (2003). See also: A. Casoli, M. Brendle, J. Schultz, A. Philippe and G. Reiter, Langmuir 17, 388 (2001).

[7] K.A. Grosch, Proc. R. Soc. London, A274, 21 (1963); K. A. Grosch, in The Physics of Tire Traction: Theory and
Experiment, D. F. Hays, A. L. Browne, Eds. (Plenum press, New York-London 1974), page 143.

[8] T. Baumberger and C. Caroli, Advances in Physics 55, 279 (2006); T. Baumberger, C. Caroli and O. Ronsin, Eur. Phys. JE11, 85 (2003); O. Ronsin and K.L. Coeyrehourcq, Proceedings of the Royal Society of London series A-Mathematical physical and engineering sciences 457, 1277 (2010).

[9] B.N.J. Persson, J. Chem. Phys. 115, 3840 (2001); B.N.J. Persson, J. Phys. Condens. Matter. 18, 7789 (2006); B.N.J. Persson and A.I. Volokitin, Phys. Rev. B65, 134106 (2002); B.N.J. Persson, Surface Science 401, 445 (1998).

[10] M. Klüppel, G. Heinrich, Rubber Chem. Technol. 73, 578 (2000); A. Le Gal and M. Klüppel, J. Chem. Phys. 123, 014704 (2005).

[11] R.F. Salant, N. Maser and B. Yang, Journal of Tribology 129, 91 (2007).

[12] B.N.J. Persson, O. Albohr, U. Tartaglino and E. Tosatti, J. Phys. Condens. Matter 17, R1 (2005).

[13] K.L. Johnson, Contact Mechanics, Cambridge University Press, Cambridge (1985).

[14] N. Patir and H.S. Cheng, J. Lubrication Technology 100, 12 (1978).

[15] B. Zappone, K.J. Rosenberg and J. Israelachvili, Tribology Letters 26, 191 (2007).

[16] For a self affine fractal surface the ratio between the height $h(\lambda)$ and wavelength $\lambda$ of the surface roughness component with wave vector $q=2 \pi / \lambda$ is $h / \lambda \sim \lambda^{2-D_{\mathrm{f}}}$. Thus the larger the fractal dimension $D_{\mathrm{f}}>2$, the faster the ratio $h / \lambda$ and will increase as the wavelength decreases, and this will tend to increase the importance of the short wavelength roughness.

[17] See, e.g., B.N.J. Persson, Sliding Friction: Physical Principles and Application, 2nd ed.; Springer: Heidelberg, 2000.

[18] B.N.J. Persson and F. Mugele, J. Phys. Condens. Matter 16, R295 (2004); B.N.J. Persson, U. Tartaglino, O. Albohr and E. Tosatti, Nature Materials 3, 882 (2004).

[19] B.N.J. Persson and C. Yang, to be published.

[20] See, e.g., B.N.J. Persson, Surface Science Reports 61, 201 (2006).

[21] P.P. Goodwyn, A. Peressadko, H. Schwarz, V. Kastner and S. Gorb, J. Comp. Physiol. A192, 1233 (2006).

[22] B.N.J. Persson, J. Phys. Condens. Matter 19, 376110 (2007).

[23] W. Federle, W.J.P. Barnes, W. Baumgartner, P. Drechsler and J.M. Smith, J. R. Soc. Interfaces 3, 689 (2006). 